\title{
A novel merged beams apparatus to study anion-neutral reactions
}

\author{
H. Bruhns, ${ }^{1, a)}$ H. Kreckel, ${ }^{1, b)}$ K. Miller, ${ }^{1}$ M. Lestinsky, ${ }^{1}$ B. Seredyuk, ${ }^{1, c)}$ W. Mitthumsiri, ${ }^{1, d)}$ \\ B. L. Schmitt, ${ }^{1, e)}$ M. Schnell, ${ }^{1, f)}$ X. Urbain, ${ }^{2}$ M. L. Rappaport, ${ }^{3}$ C. C. Havener, ${ }^{4}$ and \\ D. W. Savin ${ }^{1}$ \\ ${ }^{1}$ Columbia Astrophysics Laboratory, Columbia University, New York, New York 10027-6601, USA \\ ${ }^{2}$ Department of Physics/PAMO, Université Catholique de Louvain, Louvain-la-Neuve B-1348, Belgium \\ ${ }^{3}$ Department of Particle Physics, Weizmann Institute of Science, Rehovot 76100, Israel \\ ${ }^{4}$ Physics Division, Oak Ridge National Laboratory, Oak Ridge, Tennessee 37831-6372, USA
}

(Received 29 October 2009; accepted 8 December 2009; published online 29 January 2010)

\begin{abstract}
We have developed a novel laboratory instrument for studying gas phase, anion-neutral chemistry. To the best of our knowledge, this is the first such apparatus which uses fast merged beams to investigate anion-neutral chemical reactions. As proof-of-principle we have detected the associative detachment reaction $\mathrm{H}^{-}+\mathrm{H} \rightarrow \mathrm{H}_{2}+\mathrm{e}^{-}$. Here we describe the apparatus in detail and discuss related technical and experimental issues. (C) 2010 American Institute of Physics. [doi:10.1063/1.3280227]
\end{abstract}

\section{INTRODUCTION}

Gas phase, anion-neutral chemistry is important for a wide range of disciplines. $\mathrm{H}^{-}$ion sources are used to produce neutral beams for spallation neutron sources, ${ }^{1}$ for high energy physics, ${ }^{2,3}$ and for heating in magnetic fusion. ${ }^{4,5}$ Gasphase reactions involving $\mathrm{H}^{-}$with neutrals are important for modeling the properties of these sources. ${ }^{6,7}$ In chemistry and physics, negative ion chemistry can be used to investigate reaction dynamics, probe potential energy surfaces, and study how chemical bonds are broken and new ones formed. ${ }^{8}$ Negative ions are often present in discharges used for plasma processing in the semiconductor industry. ${ }^{9-11}$ Accurate negative ion chemistry networks are important for understanding the properties of these plasmas. ${ }^{12-14} \mathrm{H}^{-}$chemistry in the early universe plays an important role in the collapse of primordial gas clouds and the formation of first stars and protogalaxies ${ }^{15-17}$ Uncertainties in this primordial chemistry limit our ability to model reliably this epoch of the universe. ${ }^{18,19}$ The negative ion chemistry of the Earth's atmosphere and other planetary atmospheres is both complex and poorly understood. ${ }^{20-23}$ Lastly, the recent discovery of anions in interstellar clouds ${ }^{24-27}$ dramatically alters our knowledge of interstellar chemistry and emphasizes the need for a better understanding of negative ion chemistry. ${ }^{28-30}$

Over the past 40 years a number of techniques have been developed and used to study anion-neutral chemistry. These methods include flowing afterglow and its successors, ${ }^{31-33}$ as well as ion traps, ${ }^{34,35}$ crossed beams, ${ }^{8,36,37}$ and guided ion

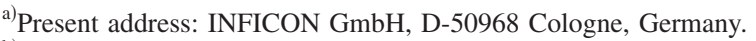

${ }^{b)}$ Present address: Department of Chemistry and Astronomy, University of Illinois at Urbana-Champaign, Urbana, IL 61801, USA.

${ }^{c)}$ Present address: Department of Physics, Ivan Franko National University, L'viv, UA-79005, Ukraine.

${ }^{d)}$ Present address: Department of Physics, Stanford University, Stanford, CA 94305-4060, USA.

${ }^{e}$ Present address: Department of Physics and Astronomy, University of Pennsylvania, Philadelphia, PA 19104-6396 USA.

${ }^{\mathrm{f}}$ Present address: Carl Zeiss NTS GmbH, D-73447 Oberkochen, Germany.
}

beams. ${ }^{38,39}$ A wide range of anion-neutral reactions have been studied using these techniques and much has been learned. Taken together, these various laboratory techniques complement one another. Each method, however, has its limitations.

Flowing afterglow and its successors are the most common methods for studying anion-neutral chemistry. They measure reaction rate coefficients versus temperature or mean thermal energy of the reactants. For collisions involving neutral atoms such as $\mathrm{H}$ and $\mathrm{O}$, uncertainties are typically at least a factor of two due to the difficulties of measuring the neutral density in the apparatus. ${ }^{40,41}$ These methods also cannot be used to study reactions with $\mathrm{C}$ which, due to its high reactivity, cannot be generated in sufficient quantities in the flows. ${ }^{40,42,43}$ Lastly, the measured rate coefficients can vary by orders of magnitude as a function of parent and buffer gas pressures. ${ }^{44}$ Thus a systematic study of rate coefficient versus these gas pressures may be necessary to generate reliable measurements. Unfortunately, such studies are rarely performed.

Ion traps measure the stored reactant and product anion populations versus time and neutral gas pressure so as to derive reaction rate coefficients. Studies are carried out at the effective temperature of the trapped ions and have been restricted to using noble and stable molecular gases. ${ }^{34,35,45}$ This excludes highly reactive atoms such as $\mathrm{H}, \mathrm{C}$, and $\mathrm{O}$, though recent work with cations suggests that such studies may be possible with $\mathrm{H}^{46}$

In crossed beam studies, the anions are crossed with a beam of neutrals produced using an effusive gas source. With the technology currently available, it is not possible to determine the spatial variation of the particle density in the neutral beam due to the slow speed of the neutrals. Hence, the overlap of the two beams cannot be reliably determined and this technique produces only relative cross section results. ${ }^{8,36,37}$

Guided ion beams send anions through a target gas cell of known density and measure the product anions. Laboratory beam energies are in the $\sim \mathrm{meV}$ to $\mathrm{eV}$ range. This 
method can produce absolute cross sections as a function of collision energy ${ }^{47,48}$ but all measurements to date have been restricted to noble and molecular gases which are chemically stable enough to store in the gas cell. ${ }^{38,39}$ This again excludes studies with atoms such as $\mathrm{H}, \mathrm{C}$, and $\mathrm{O}$.

Given the limitations of the above approaches, there is clearly a need for new methods to study anion-neutral chemistry. To this end, we have adapted the merged beams principle to study these reactions (see Refs. 49-52 for a history and overview of the merged beams method). As far as we are aware, our work here is the first to use merged beams for studying anion-neutral chemistry. Using fast beams allows us to utilize standard laboratory techniques to determine beam shapes and overlaps. ${ }^{51,53,54}$

The proof-of-principle detection reported here is the associative detachment $(\mathrm{AD})$ reaction

$$
\mathrm{H}^{-}+\mathrm{H} \rightarrow \mathrm{H}_{2}+\mathrm{e}^{-} .
$$

Beginning with a fast $\mathrm{H}^{-}$anion beam, we use a laser to photodetach and neutralize a portion of the $\mathrm{H}^{-}$beam, thereby creating a self merged, anion-neutral beams arrangement. Because the beams copropagate with minimal divergences, center-of-mass energies down to the $\mathrm{meV}$ range can be achieved. This new approach is well suited for chemical studies with systems such as $\mathrm{H}, \mathrm{C}$, and $\mathrm{O}$ which can all be generated via photodetachment of a parent atomic anion beam.

The remainder of the paper is organized as follows: Sec. II describes our experimental approach and Sec. III gives a detailed description of our novel apparatus. Background suppression is discussed in Sec. IV. Signal determination and detection of the $\mathrm{AD}$ process are presented in Sec. V. We conclude with a short summary in Sec. VI.

\section{EXPERIMENTAL APPROACH}

The relative energy $E_{r}$ between two beams $i=\{1,2\}$ is given by ${ }^{49}$

$$
E_{r}=\mu\left(\frac{E_{1}}{m_{1}}+\frac{E_{2}}{m_{2}}-2 \sqrt{\frac{E_{1} E_{2}}{m_{1} m_{2}}} \cos \theta\right),
$$

where $E_{i}$ is the kinetic energy, $m_{i}$ is the particle mass, $\mu=m_{1} m_{2} /\left(m_{1}+m_{2}\right)$ is the reduced mass, and $\theta$ is the intersection angle. Here, we take the $\mathrm{H}^{-}$anion beam as beam 1, and for beam 2 the neutral $\mathrm{H}$ beam which is created via photodetachment of the $\mathrm{H}^{-}$parent beam.

In order to set the collision energy, the photodetachment takes place in a floating cell that can be biased to an arbitrary potential $U_{\mathrm{f}}$. Upon entering the floating cell, the $\mathrm{H}^{-}$beam energy becomes $E_{1, \mathrm{f}}=E_{1}+e U_{\mathrm{f}}$, where $e$ is the elementary charge. Part of the $\mathrm{H}^{-}$beam is photodetached in the central portion of the floating cell. After photodetachment, the part of the $\mathrm{H}^{-}$beam unaffected by the laser exits the floating cell and returns to its initial energy $E_{1}$. The photodetached $\mathrm{H}$, however, is unaffected by the change in the electrostatic potentials and maintains the energy that the parent $\mathrm{H}^{-}$beam had in the floating cell giving $E_{2}=E_{1}+e U_{\mathrm{f}}$. Though the beams have laboratory energies on the order of $10 \mathrm{keV}$, this self-merged beams approach will allow us to achieve relative energies as low as a few $\mathrm{meV}$, limited largely by the divergences of the beams.

As the two $\sim 10 \mathrm{keV}$ beams copropagate ballistically, they interact with one another. The resulting AD-generated $\mathrm{H}_{2}$ molecules have a laboratory energy which is essentially the sum of the energy for the $\mathrm{H}$ and $\mathrm{H}^{-}$parent beams $E_{\mathrm{H}_{2}}=E_{1}+E_{2} \sim 20 \mathrm{keV}$ (see Appendix for a more exact derivation). Here, we have neglected the $3.75 \mathrm{eV}$ released in reaction 1 which is shared between the internal energy of the $\mathrm{H}_{2}$ molecule and the kinetic energy of the detached electron. ${ }^{55}$ Since the reaction frame is moving at high velocities in the laboratory frame, the angular spread of the product $\mathrm{H}_{2}$ molecules is strongly compressed in the forward direction. $^{51,53}$

A major challenge to using merged beams to measure reaction 1 is which of the end products to detect and how. We initially carried out a series of modeling studies investigating the feasibility of detecting the AD-generated electrons. However, the parent $\mathrm{H}^{-}$beam can collide with residual gas in the system and undergo electron detachment. We estimated that in order to reduce the resulting electron background to manageable levels required pressures on the order of $\sim 10^{-11}$ Torr or lower. Also, fast particle beams produce background electrons when the beams pass close to surfaces inside the vacuum system. Additionally, electron emission from voltages applied to electrodes inside the vacuum system can produce background electrons. Taken together these issues all made detecting the $\mathrm{AD}$-generated electrons a most unpromising approach.

Directly detecting the $\mathrm{H}_{2}$ is also not feasible. While the $\mathrm{H}^{-}$beam can be readily separated from the $\mathrm{H}_{2}$ and $\mathrm{H}$, no realistic beam optics method exists to separate the neutral $\mathrm{H}_{2}$ product from the $\sim 10^{9}$ times more intense parent $\mathrm{H}$. One could potentially use the differing energies of the $\mathrm{H}_{2}$ and $\mathrm{H}$. However, the $\mathrm{H}$ beam particle currents needed to get usable signal rates would require an energy analyzing, singleparticle-counting detector which could handle count rates of $\sim 10^{11} \mathrm{~s}^{-1}$. We are unaware of any such detectors currently available.

In the end we had the novel idea of indirectly detecting the AD-generated $\mathrm{H}_{2}$. After the interaction region, the $\mathrm{H}^{-}$ parent beam is deflected into a Faraday cup. The neutral $\mathrm{H}$ beam and the $\mathrm{AD}$-generated $\mathrm{H}_{2}$ molecules continue traveling ballistically into and through a gas cell. Some of the fast $\mathrm{H}_{2}$ molecules are stripped via the reaction

$$
\mathrm{H}_{2}+\mathrm{X} \rightarrow \mathrm{H}_{2}^{+}+\left[\mathrm{X}, \mathrm{e}^{-}\right],
$$

where $\mathrm{X}$ is the stripping gas. The final states of the stripping gas and the ionized electron are both unimportant. This process converts the $\mathrm{H}_{2}$ into $\sim 20 \mathrm{keV} \mathrm{H}_{2}^{+}$which we subsequently detect. Reactions in the gas cell also produce $\mathrm{H}^{+}$ through stripping of the $\mathrm{H}$ beam and through dissociative ionization of the $\mathrm{H}_{2}$. The energy of the resulting $\mathrm{H}^{+}$is approximately $E_{\mathrm{H}_{2}} / 2 \sim 10 \mathrm{keV}$ which allows us to readily suppress this background using energy analyzers.

After the gas cell, the neutral and charged particle beams enter a series of two electrostatic deflectors which is used to selectively direct particles of energy $E_{\mathrm{H}_{2}}$ (i.e., the $\mathrm{H}_{2}^{+}$ions) 


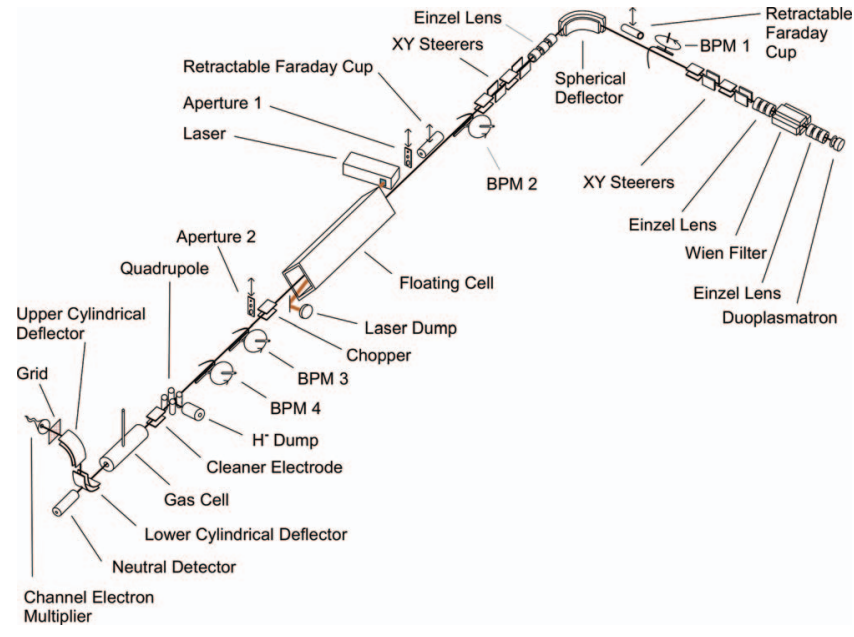

FIG. 1. (Color) Schematic overview of the apparatus. The first leg from the duoplasmatron to the spherical deflector is $\sim 2 \mathrm{~m}$ in length. The second leg from the spherical deflector to the neutral detector is $\sim 7 \mathrm{~m}$. The individual elements are not to scale. BPM is short for beam profile monitor. Turbomolecular pumps (TMPs) are located at the duoplasmatron, the spherical deflector, the center of the photodetachment chamber, the quadrupole, and the lower cylindrical deflector (LCD). Additional details are given in the text.

into a channel electron multiplier (CEM) particle counter. This allows us to reliably detect the AD-generated $\mathrm{H}_{2}$ molecules with excellent background suppression. Additional backgrounds are determined and corrected for by chopping both parent beams on and off out of phase with one another. ${ }^{53,56,57}$ By measuring the $\mathrm{H}^{-}$and $\mathrm{H}$ beam currents, the overlap of the two beams, and the $\mathrm{H}_{2}^{+}$signal rate, we can perform relative measurements for reaction 1 as a function of center-of-mass collision energy. Determining the gas cell column density and using the $\mathrm{H}_{2}$ to $\mathrm{H}_{2}^{+}$stripping cross section, we can turn our relative results into absolute measurements.

\section{APPARATUS DESCRIPTION}

\section{A. Beam preparation}

An overview of the apparatus is given in Fig. 1. It begins with a Peabody Scientific Duoplasmatron Ion Source System using $\mathrm{H}_{2}$ gas. This is pumped by a turbomolecular pump (TMP) with a pumping speed of $450 \mathrm{l} \mathrm{s}^{-1}$ for $\mathrm{H}_{2}$. The duoplasmatron is followed by an einzel lens and then a Wien filter. We use the ion source to generate $\mathrm{H}^{-}$ions and accelerate them to a laboratory energy of typically $10 \mathrm{keV}$. The einzel lens focuses the beam onto the exit of the Wien filter. The crossed magnetic and electric field configuration of the Wien filter allows us to purify the $\mathrm{H}^{-}$beam. The charge-tomass selection of the Wien filter prevents transmission of electrons, $\mathrm{O}^{-}$, and any other negative ions emitted by the source.

All the subsequent components of the ion optics system were designed and built in-house. A second einzel lens at the exit of the Wien filter is used to make the $\mathrm{H}^{-}$beam parallel. This is followed about $200 \mathrm{~mm}$ later by an $\sim 270 \mathrm{~mm}$ long set of XY steerers consisting of four alternating pairs of parallel plates which steer in the horizontal (X) and vertical (Y) directions. Each pair of opposing plates can be used to change the beam angle by applying a voltage between the plates. Alternatively, by biasing opposing plates with the same electric potential with respect to ground, each plate pair can be used as an one-dimensional (1D) lens. Because electric fields superimpose, both steering and lensing effects can be obtained simultaneously by applying the sum voltages of the steering and lensing fields. Thus, two pairs of vertical plates and two pairs of horizontal plates give full control over the beam direction, including parallel shifts, and also allow for beam shaping.

About $460 \mathrm{~mm}$ downstream from the exit of the steerers we use a rotating wire scanner beam profile monitor (BPM 1) to determine the beam shape. The ion current is measured about $230 \mathrm{~mm}$ later using a retractable Faraday cup. We can tune the source and ion optics to deliver up to $18 \mu \mathrm{A}$ of $\mathrm{H}^{-}$ current at this point. With the beam tuned for optimum transport through the entire system, we typically find about $14 \mu \mathrm{A}$ at this point. Note that here and throughout the rest of this paper all $\mathrm{H}^{-}$and $\mathrm{H}$ currents are for $10 \mathrm{keV}$ beams unless otherwise specified.

With this first Faraday cup removed from the beam path, the beam travels about $160 \mathrm{~mm}$ and enters a spherical deflector ${ }^{58-63}$ which is pumped by a TMP with pumping speeds of $380 \mathrm{l} \mathrm{s}^{-1}$ for $\mathrm{H}_{2}$ and $400 \mathrm{l} \mathrm{s}^{-1}$ for He. The base pressure at this point is $\sim 4 \times 10^{-8}$ Torr, using the manufacturer given $\mathrm{H}_{2}$ scale factor for the pressure gauge. During operation, $\mathrm{H}_{2}$ from the ion source is the dominant gas load at this point with a smaller contribution of He coming from the gas cell described in Sec. III D. Typical operating background pressures are $\sim 1 \times 10^{-7}$ Torr.

The spherical deflector bends the beam by $90^{\circ}$ along a radius of curvature of about $150 \mathrm{~mm}$. This prevents a direct line of sight from the source to the interaction region so that UV photons and neutrals that leave the source do not alter the reactions studied. Since the spherical deflector focuses the beam at its exit, a third einzel lens, located shortly after the exit, is used to make the beam parallel again. This is followed by a set of XY steerers identical to the first, BPM 2 about $610 \mathrm{~mm}$ from the exit of the deflector, and a retractable Faraday cup about $840 \mathrm{~mm}$ from the exit. We can tune the beam to the same current here as measured in the first Faraday cup. However, with the beam tuned for optimum transport throughout the entire length of the apparatus, we typically have $8 \mu \mathrm{A}$ left at this point.

About $1055 \mathrm{~mm}$ after the spherical deflector exit there is an aperture plate mounted on a linear manipulator (aperture 1). The plate has three circular openings with diameters of 5 , 7 , and $10 \mathrm{~mm}$, each of which can be inserted in the center of the beamline. The plate can also be fully retracted. The center of the photodetachment chamber (see Sec. III B) is located $1410 \mathrm{~mm}$ after this aperture. An identical aperture plate on a manipulator arm (aperture 2) is located $1384 \mathrm{~mm}$ further downstream from the center of the photodetachment chamber.

The two aperture plates allow us to define the beam, reduce background, and limit the beam divergence. The distance between the two aperture plates is $2794 \mathrm{~mm}$. Reducing the beam divergence with the aperture plates allows us to reach collision energies on the order of a few meV. The measurements described here were conducted using both 5 
$\mathrm{mm}$ apertures, resulting in a half angle beam divergence of $1.79 \mathrm{mrad}$ or $0.1^{\circ}$. Due to the small acceptance of this setup, the resulting $\mathrm{H}^{-}$beam current at the end of the $\mathrm{H}^{-}$beam path is reduced to typical values of $0.6 \mu \mathrm{A}$. Using both $7 \mathrm{~mm}$ apertures we typically transmit currents of $1.1 \mu \mathrm{A}$, and with both $10 \mathrm{~mm}$ apertures of $3 \mu \mathrm{A}$.

Outside of the vacuum vessel, rectangular magnetic field coils extending from the second Faraday cup to the entrance of the analyzer (see Sec. III E) are mounted in the horizontal and vertical planes parallel to the ion beam direction. These coils cancel the corresponding effects of the Earth's magnetic field, reducing the respective fields by roughly an order of magnitude. This in turn reduces to insignificant the deflection of the $\mathrm{H}^{-}$beam and the signal $\mathrm{H}_{2}^{+}$ions.

\section{B. Photodetachment region}

The photodetachment (PD) chamber begins $1486 \mathrm{~mm}$ after the exit of the spherical deflector. The PD chamber is $1930 \mathrm{~mm}$ in length. The PD laser beam is overlapped with the $\mathrm{H}^{-}$beam at an intersection angle of $2.7^{\circ}$ in order to increase the overlap length and thereby enhance the photodetachment efficiency. To achieve this small angle, the PD laser beam travels almost parallel to the $\mathrm{H}^{-}$beam and must be coupled into and out of the vacuum system through viewports close to the centerline of the $\mathrm{H}^{-}$beam. This was achieved using $16 \mathrm{~mm}$ diameter beam pipe leading into the entrance and coming out of the exit of the PD chamber. An advantage of using such narrow pipe is that it leads to strong differential pumping of the chamber. The dominant partial pressure in the PD vessel during measurement stems from He emanating from the gas cell described in Sec. III D. The PD chamber is pumped by a TMP with pumping speeds of $510 \mathrm{l} \mathrm{s}^{-1}$ for $\mathrm{H}_{2}$ and $600 \mathrm{l} \mathrm{s}^{-1}$ for $\mathrm{He}$. The base pressure in the chamber is $\sim 2 \times 10^{-8}$ Torr, using the provided He gas scale factor. During measurement, typical operating background pressures in the chamber are $\sim 5 \times 10^{-7}$ Torr.

Within the PD chamber is a floating cell of $1200 \mathrm{~mm}$ in length, the center of which is aligned with the center of the chamber, nearly midway between the two beam-defining apertures. Photodetachment occurs within this floating cell, the length of which extends well beyond the overlap region of the laser and ion beam so as to have no potential gradient where the PD occurs [as determined by SIMION $^{\circledR}$ (Ref. 64) modeling].

Using a DILAS Diode Laser Inc. diode stack to generate a laser beam at $975 \mathrm{~nm}(1.27 \mathrm{eV})$ with $\sim 1.4 \mathrm{~kW}$ power, we can create a beam of ground state atomic $\mathrm{H}$ via photodetachment of the $\mathrm{H}^{-}$parent beam. This is a major advantage compared to creating the $\mathrm{H}$ beam through single electron detachment off a gas, a process which produces both ground and excited states of $\mathrm{H}^{65}$ The laser diodes are driven by an AMTRON GmbH power supply that allows switching the diodes on and off within $25 \mu \mathrm{s}$. This provides us with the means to chop the $\mathrm{H}$ beam on and off.

The laser is brought to a focus near the center of the floating cell. The focus calculated by DILAS is nearly Gaussian in shape with full width at half maximum widths of 7 and $4 \mathrm{~mm}$ in horizontal and vertical direction, respectively. Using these shapes and the predicted $\mathrm{H}^{-}$beam profile in the center of the floating cell, we estimate a photodetachment efficiency of $f=7.4 \%$. Additional details concerning the determination of $f$ and the measurement of the neutral beam particle current will be given in a future publication.

\section{Interaction region}

The beginning of the interaction region is defined by an electrostatic plate which is used to deflect the $\mathrm{H}^{-}$beam. This so-called "chopper" plate is located at a distance of $292 \mathrm{~mm}$ after the end of the PD chamber and is also $127 \mathrm{~mm}$ before aperture 2 or $1257 \mathrm{~mm}$ after the center of the PD region. With no voltage on this plate, the $\mathrm{H}^{-}$beam continues into the interaction region where it can react with the $\mathrm{H}$ beam. Applying a voltage to this plate allows us to demerge the $\mathrm{H}^{-}$ from the $\mathrm{H}$ beam, thereby turning off beam-beam reactions in the interaction region. The voltage on this chopper is controlled using a Behlke Power Electronics GmbH high voltage switch with a switching time of better than $100 \mathrm{~ns}$. As mentioned earlier, the atomic $\mathrm{H}$ beam is chopped on and off further upstream from this point by switching the laser beam on and off, respectively. Chopping of the two beams allows us to determine the various backgrounds in the measurement and to extract the signal from the combined signal plus backgrounds (see Sec. V).

The beam-beam overlap is measured in the interaction region in two separate locations. BPMs 3 and 4 are used, located at respective distances of 305 and $762 \mathrm{~mm}$ from the chopper. The overlap determination using the BPMs is described in detail in Ref. 66 for double wire BPMs but is also appropriate for the single wire BMPs used here.

At a distance of $990 \mathrm{~mm}$ after the $\mathrm{H}^{-}$chopper, an electrostatic quadrupole demerges the $\mathrm{H}^{-}$beam from the $\mathrm{H}$ beam, deflecting the anion beam into a Faraday cup (hereafter, the $\mathrm{H}^{-}$dump). The quadrupole marks the end of the interaction region. It also removes any other charged particles from the merged beams, such as $\mathrm{H}^{+}$and $\mathrm{H}_{2}^{+}$ produced from collisions with the residual gas upstream of the quadrupole. Typical voltages on the quadrupole of $\left|U_{q}\right| \sim 7.3 \mathrm{kV}$ are used.

Within the quadrupole, collisions of $\mathrm{H}$ and $\mathrm{H}_{2}$ with the residual gas can generate protons. We found that such collisions can lead to additional background. If these protons are created near the downstream positive electrode of the quadrupole, they can be accelerated out of the quadrupole, almost in the direction of the neutral beam, and into the analyzer. Their maximum energy is approximately $10 \mathrm{keV}+e U_{q}$ $\sim 17 \mathrm{keV}$. This is close enough to the $\sim 20 \mathrm{keV}$ energy of the $\mathrm{H}_{2}^{+}$signal ions that they make it sufficiently far into the analyzer and near enough to the CEM so as to produce a significant background, most likely due to a mixture of particles and photons. To eliminate this background, we have mounted a cleaning electrode between the quadrupole exit and the gas cell entrance and applied a $+2 \mathrm{kV}$ potential, thereby directing into the vacuum chamber wall charged particles created between the quadrupole and the gas cell. As a result, the only particles which can enter the gas cell are those of the neutral parent $\mathrm{H}$ beam $(\sim 50 \mathrm{nA}$ particle current or $\sim 3 \times 10^{11}$ particles $\mathrm{s}^{-1}$ ) and the $\sim 100 \mathrm{~s}^{-1} \mathrm{AD}$-generated $\mathrm{H}_{2}$ molecules. 
The interaction region is pumped by a TMP with speeds of $510 \mathrm{l} \mathrm{s}^{-1}$ for $\mathrm{H}_{2}$ and $600 \mathrm{l} \mathrm{s}^{-1}$ for He. The dominant gas load in the interaction region is He flowing out of the gas cell. Using the provided $\mathrm{He}$ gas scale factor for the pressure gauge, the base pressure in the interaction region is $\sim 5 \times 10^{-9}$ Torr. Typical operating background pressures here during measurement are $\sim 3 \times 10^{-5}$ Torr. Modeling using the vacuum tracking code VAKLOOP, ${ }^{67,68}$ used to design the vacuum system, indicates this pressure begins roughly in the short $16 \mathrm{~mm}$ diameter beam pipe at the exit of the PD chamber.

\section{Gas cell}

After passing through the quadrupole, the fast $\mathrm{H}$ and $\mathrm{H}_{2}$ enter a gas cell of $787 \mathrm{~mm}$ in length. The entrance aperture is circular with a diameter of $16.0 \mathrm{~mm}$. Helium is fed into the gas cell through a leak valve. A He pressure of $\sim 2 \times 10^{-4}$ Torr is typically used for measurements. The base pressure with no helium fed in is $\sim 3 \times 10^{-7}$ Torr. The pressures were measured using the $\mathrm{He}$ gas scale factor provided for the pressure gauge. The exit aperture of the gas cell is $15.24 \mathrm{~mm}$ wide and $12.70 \mathrm{~mm}$ high. Following the gas cell comes the analyzer (Sec. III E) which is also differentially pumped. The VAKLOOP pressure profile simulation of the entire apparatus gave results that agree well with the measured pressures in the interaction and analyzer regions.

Within the gas cell, some of the AD-generated fast $\mathrm{H}_{2}$ strips on the helium atoms, producing fast $\mathrm{H}_{2}^{+}$. The efficiency for converting AD-generated $\mathrm{H}_{2}$ into $\mathrm{H}_{2}^{+}$depends on the stripping cross section $\sigma_{\mathrm{st}}$ and the helium column density (i.e., target thickness)

$$
N_{\mathrm{He}}=\int n_{\mathrm{He}}(\ell) d \ell,
$$

where $n_{\mathrm{He}}(\ell)$ is the helium number density along the beam path and $d \ell$ the differential path length. For the low target thicknesses that we operate at (often called the linear regime) the rate for $\mathrm{H}_{2}^{+}$formation is given by

$$
R_{\mathrm{H}_{2}^{+}}=R_{\mathrm{H}_{2}} \sigma_{\mathrm{st}} N_{\mathrm{He}}
$$

where $R_{\mathrm{H}_{2}}$ is the rate at which AD-generated $\mathrm{H}_{2}$ molecules enter the gas cell and $\sigma_{\mathrm{st}}=1.04 \times 10^{-16} \mathrm{~cm}^{2}$ for $20 \mathrm{keV} \mathrm{H}$ on He. ${ }^{69}$ Using the gas cell length and pressure and the stripping cross section, we estimate the efficiency for converting $\mathrm{H}_{2}$ into $\mathrm{H}_{2}^{+}$to be $\sim 5 \%$. Thus for $R_{\mathrm{H}_{2}} \sim 100 \mathrm{~s}^{-1}$ we expect $R_{\mathrm{H}_{2}^{+}} \sim 5 \mathrm{~s}^{-1}$.

For relative cross section measurements one does not need to know the precise helium column density in the gas cell. It is sufficient that $N_{\mathrm{He}}$ varies linearly with the gas cell pressure reading. In fact, our measurements show that the pressure in the interaction region and the analyzer scale linearly with the measured gas cell pressure. Hence, relative measurements can be carried out by normalizing the results of each data run to the corresponding pressure reading for the gas cell. Absolute measurements require a determination of $N_{\mathrm{He}}$ as will be described in a future publication.

\section{E. Analyzer and detection}

The $\mathrm{H}$ and $\mathrm{H}_{2}$, along with any $\mathrm{H}_{2}^{+}$and $\mathrm{H}^{+}$formed in the gas cell, all enter the analyzer which comes immediately after the gas cell exit. A TMP with pumping speeds of $4501 \mathrm{~s}^{-1}$ for $\mathrm{H}_{2}$ and $520 \mathrm{l} \mathrm{s}^{-1}$ for $\mathrm{He}$ is used on this portion of the apparatus. The dominant gas load here is He flowing from the gas cell. Using the provided $\mathrm{He}$ gas scale factor for the pressure gauge, the base pressure was $\sim 3 \times 10^{-8}$ Torr. During measurement, typical operating background pressures in the analyzer are $\sim 3 \times 10^{-5}$ Torr.

The analyzer consists of two consecutive electrostatic cylindrical $90^{\circ}$ deflectors. The $\sim 20 \mathrm{keV} \mathrm{H}_{2}^{+}$signal is separated from the $\sim 10 \mathrm{keV} \mathrm{H}^{+}$background (cf. Sec. II) using the properties of electrostatic analyzers which discriminate charged particles based on their energy. The first or lower cylindrical deflector (LCD) bends the $\mathrm{H}_{2}^{+}$ions upwards. The second or upper cylindrical deflector (UCD) bends them back to fly horizontal but perpendicular to the original beam axis. The nominal radius of curvature for the trajectory of the beam in each deflector is $159 \mathrm{~mm}$. The LCD has a hole in the outer plate to allow the neutral beam to pass through.

Both cylindrical deflectors have been constructed utilizing a novel configuration which incorporates cylindrical plates of different heights. ${ }^{70}$ Standard cylindrical deflectors (sometimes referred to as radial cylindrical analyzers) are configured using plates of the same height. Such an arrangement focuses the exiting beam only in the plane of curvature but provides no control of the shape of the beam in the perpendicular direction. ${ }^{63,71}$ With our novel arrangement, in the plane of curvature the electric fields exert the same force on the ions as in an ordinary cylindrical deflector. However, the inhomogeneity of the fields caused by the height difference of the plates provides focusing properties in the direction perpendicular to the plane of curvature. This double-focusing radial cylindrical deflector functions much as a spherical deflector does. But based on our experience in designing, constructing, and mounting the spherical deflector described in Sec. III A, we find these cylindrical deflectors to be much easier and more cost-effective to manufacture and install than a spherical deflector.

After exiting the $\mathrm{UCD}$, the signal $\mathrm{H}_{2}^{+}$ions are detected using a CEM particle counter, from Dr. Sjuts Optotechnik $\mathrm{GmbH}$, with a circular opening of $25 \mathrm{~mm}$. The entrance funnel of the CEM is grounded while $+2 \mathrm{kV}$ is applied to the tail end. The signal is capacitively coupled out. A grid with a geometric transmittance of $T_{g}=90 \%$ is mounted in front of the CEM and a voltage of $-300 \mathrm{~V}$ applied to the grid. This voltage helps to repel electrons generated in the analyzer due to the impact of the $\mathrm{H}^{+}$created in the gas cell. It also helps to redirect any outward moving electrons produced by $\mathrm{H}_{2}^{+}$ions striking the CEM cone, sending them back into the CEM where they can generate an electron cascade giving us the signal we detect. The CEM output pulses are preamplified and then sent to a timing filter amplifier. The measured pulse height distribution (PHD) is well above the noise level. We set the discriminator level to lie above the noise and below the signal PHD. For these conditions the CEM detection efficiency $\eta$ for incident particles at energies of greater than 3 $\mathrm{keV} / \mathrm{u}$ (where $\mathrm{u}$ is the atomic mass unit) is expected to be 
near unity. ${ }^{72,73}$ Here the $\mathrm{H}_{2}^{+}$ions travel at $10 \mathrm{keV} / \mathrm{u}$ and combining the results of Refs. 72 and 73 we expect $\eta=0.98 \pm 0.02$.

The neutral $\mathrm{H}$ beam passes through a hole in the outer deflection plate of the LCD and continues into a neutral detector. The $\mathrm{H}$ beam particle current is monitored by measuring the secondary negative particle emission from the detector target. The neutral detector is similar to the Faraday cup design of Ref. 74 with an inner copper target and an inner cup in physical (i.e., electrical) contact with the target. The main difference here is the addition of a ring at the entrance which is set to the same voltage as the outer cup. The outer cup is called the repeller in Ref. 74. This design allows us to use the neutral detector either to collect neutrals or as a Faraday cup to measure charged beam currents. As a neutral cup, we measure the secondary particle emission current by applying a voltage difference between the inner and outer cups and measuring the charge flowing from the inner cup (the beam target) to the outer cup.

The optimal voltages for transmitting $\mathrm{H}_{2}^{+}$through the analyzer were initially determined by extracting a $20 \mathrm{nA}$ beam of $20 \mathrm{keV} \mathrm{H}_{2}^{+}$from the ion source, sending it through the system, and then using the quadrupole with the voltages set to direct the positive beam into the $\mathrm{H}^{-}$dump just before the gas cell. Electron capture off the residual gas in the second leg of the apparatus produced $20 \mathrm{keV}$ neutral $\mathrm{H}_{2}$ which we used to simulate the AD-generated signal. This neutral beam was sent into the gas cell creating (depending on the pressure) several $\mathrm{kHz}$ of simulated signal at the CEM. With such a high rate it was straightforward to scan individually the voltage on the LCD and the UCD and thereby to map out the plateau in CEM signal counts versus voltage for each deflector. Using these results as a guide, we then used the true $\mathrm{H}_{2}^{+}$signal ions to map out the plateau. As is described in Sec. V, both methods give results which agree well.

The transmittance $T_{a}$ of $\mathrm{H}_{2}^{+}$through the analyzer was determined using the full $20 \mathrm{nA} \mathrm{H}_{2}^{+}$beam as first measured in the $\mathrm{H}^{-}$dump Faraday cup using quadrupole voltages appropriate for collecting the positive beam. To measure $T_{a}$ we reconfigured the neutral detector as a Faraday cup and we also replaced the CEM with a Faraday cup. $T_{a}$ was derived by measuring the $\mathrm{H}_{2}^{+}$current in the reconfigured neutral detector (with the analyzer voltages set to $0 \mathrm{~V}$ ) and then measuring the current at the CEM position with the analyzer set to the previously determined optimum voltages. In this way we measured $T_{a}=(99 \pm 1) \%$.

\section{F. Computer control}

The apparatus is largely controlled using custom written LABVIEW software. The $\sim 30$ power supplies used for steering and tuning the ion beam from the first einzel lens through to the UCD are all computer controlled. Manual optimization of the $\mathrm{H}^{-}$or $\mathrm{H}$ currents through the system is extremely time consuming given the large dimensionality of the steering and tuning phase space, so we have written an optimization program which randomly walks its way through this phase space, changing voltages by a specified fraction, and keeping only those changes which increase the beam current.
Computer control of the systems allows us to record the settings for almost all of the power supplies. At the end of a typical day, we store all settings and at the beginning of the next operating day we recall these values and read them into the control program. This greatly speeds up our ability to return to a near optimal operating configuration of the apparatus. Manual tuning of the beam is also possible using the arbitrarily assignable digital encoders of a midi mixer as an inexpensive, adaptable beam control panel.

The floating cell voltage and the laser are also computer controlled. The voltages on the various Faraday cups, the compensation coils for the Earth's magnetic field, the neutral detector, the CEM and grid, and the duoplasmatron plasmatuning controls are all manually set.

Once the apparatus is set up for data collection, data acquisition is entirely computer controlled. The BPMs are switched off during data acquisition. The leak valve for the gas cell is manually controlled but the pressure gauge is read into the computer. The laser power, beam currents, and the CEM signal are also read into the computer. Data are collected, usually while the voltage for a specified power supply is varied. In Sec. V, we report data collected as we individually swept the LCD and UCD voltages.

\section{BACKGROUND SUPPRESSION}

Given that the expected signal $R_{\mathrm{H}_{2}^{+}}$is only $\sim 5 \mathrm{~s}^{-1}$, considerable effort was put into the suppression of background counts due to particles other than $\mathrm{H}_{2}^{+}$(including photons). Implementing all of the actions reported in this section reduced the background to insignificant levels.

The first two steps in the suppression of the non- $\mathrm{H}_{2}^{+}$ background are provided by the quadrupole and the cleaning electrode at its exit (see Sec. III C). These two prevent charged particles in the beam from passing into the gas cell, allowing only neutral particles to enter. The next source of background is the $\mathrm{H}^{+}$created through stripping of the neutral $\mathrm{H}$ beam in the gas cell. Considering the ratio of the $\mathrm{H}$ and $\mathrm{H}_{2}$ particle currents and their stripping cross sections, ${ }^{69,75}$ the $\mathrm{H}^{+}$ background is estimated to be $\sim 3 \times 10^{9}$ times larger than the $\mathrm{H}_{2}^{+}$rate. A single $90^{\circ}$ cylindrical deflector was insufficient to fully suppress this $\mathrm{H}^{+}$background and it proved necessary to use two cylindrical deflectors in series as described in Sec. III E.

Nonsignal particles and photons, which make their way through the LCD or which are generated by collisions in the LCD, can enter the UCD, strike surfaces within the deflector, and create electrons that can exit in the direction of the CEM. To prevent these electrons from entering the CEM and creating background counts, we set the grid in front of the CEM at $-300 \mathrm{~V}$ to repel electrons.

Photons striking the CEM are another source of background counts. Most of this background was suppressed by placing an aluminum sheet in between the two analyzers with a $15 \times 15 \mathrm{~mm}^{2}$ opening in the center for the $\mathrm{H}_{2}^{+}$ions to pass through. In order to prevent photons from reaching the CEM through this hole and subsequently reflecting on the stainless steel surfaces of the analyzer plates, all noninsulator 


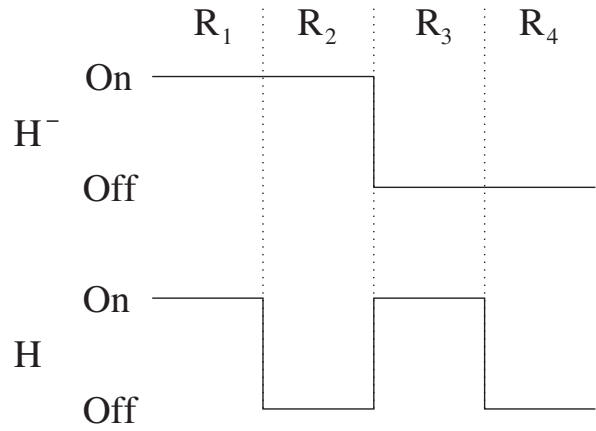

FIG. 2. Schematic of the chopping pattern used for data acquisition. The pattern indicates when the $\mathrm{H}^{-}$and $\mathrm{H}$ beams were on or off in the interaction region.

elements of the cylindrical deflectors were coated black with graphite. This was done for both the LCD and UCD.

\section{SIGNAL DETERMINATION}

Measurements are typically carried out keeping $E_{\mathrm{H}_{2}}$ constant as we vary $E_{r}$ by biasing the floating cell (see Appendix). This ensures a constant $\sigma_{\text {st }}$ for all measurements and removes any uncertainties in the conversion factor (Eq. (5)) were $E_{\mathrm{H}_{2}}$ to vary. This also allows us to keep the same analyzer voltages for all values of $E_{r}$.

\section{A. Background subtraction}

As a result of our success in suppressing the non- $\mathrm{H}_{2}^{+}$ background to the point where it is insignificant, the remaining CEM counts are essentially due only to $\mathrm{H}_{2}$ molecules formed in the second leg of the apparatus with an extremely small contribution from CEM dark counts generated by cosmic rays and electronic noise. By chopping the $\mathrm{H}^{-}$and $\mathrm{H}$ beams on and off ${ }^{53,56,57}$ as shown in Fig. 2, we can extract the desired signal rate $S$ for $\mathrm{H}_{2}$ formed in the interaction region from the background $\mathrm{H}_{2}$ formed outside this region. With this pattern we measure four different rates: both beams on $\left(R_{1}\right), \mathrm{H}^{-}$beam on and $\mathrm{H}$ beam off $\left(R_{2}\right), \mathrm{H}^{-}$beam off and $\mathrm{H}$ beam on $\left(R_{3}\right)$, and both beams off $\left(R_{4}\right)$.

The laser beam and thus the $\mathrm{H}$ beam state is switched every $5 \mathrm{~ms}$. The times required to switch from on to off and vice versa is $<25 \mu$ s. The $\mathrm{H}^{-}$beam is switched every 10 ms. The switching of the $\mathrm{H}^{-}$beam is complete within hundreds of ns. The amplified CEM pulses are discriminated, converted to NIM signals, and then, depending on the states of the two beams, are distributed by electronic logic units to one of the four different counters that are used to record rates $R_{1}$ through $R_{4}$. Since the dwell time at any given beam state is more than 100 times longer than the switching times, pulses arriving during the switching do not make any significant contribution to the measured rates.

We separate the different rates into those with the laser on $\left(R_{1}-R_{3}\right)$ and those with the laser off $\left(R_{2}-R_{4}\right)$. These differences subtract out the contributions due to any $\mathrm{H}_{2}$ produced upstream of the chopper. The differences also subtract out the dark rate $N$ which is the same for all four portions of the chopping pattern. Using standard background subtraction methods, ${ }^{53,56,57} S$ should be given by
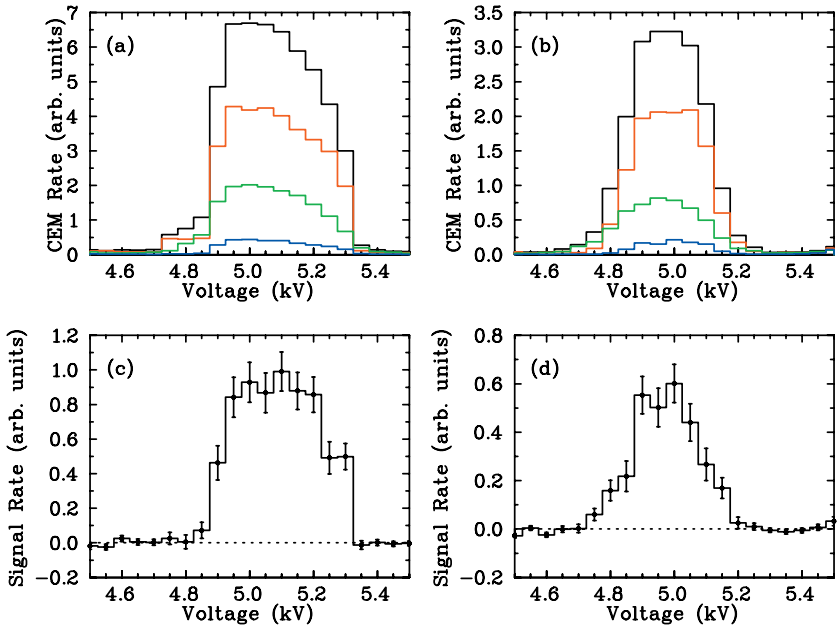

FIG. 3. (Color) CEM count rate while scanning the voltages on (a) the LCD and (b) the UCD. Going from top to bottom, the histograms are $R_{1}$ (black), $R_{3}$ (red), $R_{2}$ (green), and $R_{4}$ (blue). The extracted signal using Eq. (6) is shown in (c) for the lower deflector and in (d) for the upper. See text for additional details.

$$
S=\left(R_{1}-R_{3}\right)-\left(R_{2}-R_{4}\right) .
$$

However, this ignores the $\sim 7.4 \%$ reduction in the $\mathrm{H}^{-}$beam current when the laser is on. Thus the $\mathrm{H}^{-}$and $\mathrm{H}$ generated backgrounds are lower when the laser is on than when it is off. Extracting the true signal rate requires taking into account these background differences. Detailing the nontrivial analytic calculation of $S$ goes beyond the scope of this paper and will be described in a future publication. However, for the proof-of-principle detection reported here, it suffices to use Eq. (6).

\section{B. Analyzer voltage settings}

The optimal voltage settings for the LCD and the UCD were determined by setting up the apparatus to measure reaction 1 while using the chopping pattern shown in Fig. 2 and scanning either the LCD or UCD in $50 \mathrm{~V}$ steps. In Fig. 3 (a) we show the detected $\mathrm{H}_{2}^{+}$signal versus $\mathrm{LCD}$ voltage. The UCD was set to $4950 \mathrm{~V}$ and $U_{\mathrm{f}}$ to $-1 \mathrm{~V}$. The small negative value of $U_{\mathrm{f}}$ was used to draw away from the $\mathrm{H}^{-}$ beam the positive ions created by the beam colliding with the residual gas and thereby prevent $\mathrm{H}^{-}$self-focusing effects in the floating cell. ${ }^{76}$ Figure $3(\mathrm{~b})$ shows the same but for the UCD and with the LCD set to $5100 \mathrm{~V}$. The low baseline level for each scan indicates that the backgrounds due to particles other than $\mathrm{H}_{2}^{+}$and due to CEM dark counts are both extremely small.

In each figure, $R_{1}$ is shown by the black histogram, $R_{2}$ by the green, $R_{3}$ by the red, and $R_{4}$ by the blue. The relative amplitudes of the four counters can readily be explained. For $R_{1}$ the laser-generated $\mathrm{H}$ beam and the $\mathrm{H}^{-}$beam both travel through to the end of the interaction region. With the highest currents and longest interaction length, this gives the highest $\mathrm{H}_{2}$ production rate. For $R_{3}$ the situation is similar but only up until the chopper. With a shorter interaction length, this gives us the second highest $\mathrm{H}_{2}$ production rate. For $R_{2}$ the $\mathrm{H}^{-}$ beam travels through to the end of the interaction region but it can react only with the weak $\mathrm{H}$ beam generated via strip- 
ping of the $\mathrm{H}^{-}$beam. The situation is similar for $R_{4}$ but now the $\mathrm{H}^{-}$is deflected at the chopper and can only interact upstream of this point. Hence we find $R_{1}>R_{3}>R_{2}>R_{4}$.

We extract the signal from the measured rates using Eq. (6). The results are shown for the LCD in Fig. 3(c) and for the UCD in Fig. 3(d). The error bars give the $1 \sigma$ counting statistics for each voltage step. The signal plateau for the LCD is centered at 5100 and extends $\sim \pm 150$. For the UCD it is centered at $4950 \mathrm{~V}$ and extends $\sim \pm 75$. These results are in excellent agreement with those of the simulated $\mathrm{H}_{2}^{+}$signal described in Sec. III E.

The data shown in Fig. 3 were collected at a relative energy of $E_{r} \sim 3.7 \mathrm{meV}$. The voltage ranges in (a) and (b) were scanned 300 times in $50 \mathrm{~V}$ steps with a total of 20 steps in each scan. The integration time at each step was $5 \mathrm{~s}$ per scan, giving a total integration time of $1500 \mathrm{~s}$ per voltage. The observed $\mathrm{H}_{2}^{+}$signal rate was $\sim 5 \mathrm{~s}^{-1}$.

The presence of the plateau in the LCD data for the optimal UCD voltage and vice versa together indicates that the size of the $\mathrm{H}_{2}^{+}$beam (a) is smaller than the mouth of the CEM and (b) is smaller than all of the various apertures in the analyzer. Hence we conclude that selecting LCD and UCD voltages lying within the range corresponding to these plateaus will ensure collection of the entire $\mathrm{H}_{2}^{+}$beam.

The ratio between the beam voltage and the required deflection voltage is $\sim 4$. Thus the $\pm 75 \mathrm{~V}$ wide plateau in Fig. 3(d) indicates that the energy acceptance of the analyzer is $\sim \pm 300 \mathrm{eV}$ for a $20 \mathrm{keV}$ beam.

\section{SUMMARY}

We have designed and constructed a novel merged beams apparatus to investigate gas-phase, anion-neutral chemistry. To the best of our knowledge, our work here represents the first use of fast merged beams to study anionneutral chemical reactions. To demonstrate the proof-ofprinciple, we have detected signal from the $\mathrm{AD}$ reaction between $\mathrm{H}^{-}$and $\mathrm{H}$ forming $\mathrm{H}_{2}$. Results and discussion of our absolute measurement for this reaction will be given in a future publication.

\section{ACKNOWLEDGMENTS}

We thank M. Bannister, A. Chutjian, A. M. Covington, A. Dorn, O. Heber, A. Müller, H. B. Pedersen, S. Schippers, C. D. Schröter, and A. Wolf, for stimulating discussions. We also thank D. Thomas for his excellent machining skills and support. D.W.S. especially thanks M. Mauel for his early support which made it possible to write the initial grant that funded this research. Work at Columbia University was supported in part by the NSF Chemistry Research Instrumentation and Facilities: Instrument Development (CRIF:ID) (Grant No. CHE-0520660) and the NSF Astronomy and Astrophysics (Grant No. AST-0807436). H.B. was also supported in part by the German Academic Exchange Service DAAD. X.U. is Senior Research Associate of the F.R.S.FNRS. C.C.H. is supported by the Office of Fusion Energy Sciences and the Office of Basic Energy Sciences, U.S. DOE, (Contract No. DE-AC05-00OR22725) with UTBattelle, LLC.

\section{APPENDIX: LABORATORY ENERGY OF THE AD-GENERATED $\mathrm{H}_{2}$}

Here we develop the basic requirements for controlling the laboratory energy of the AD-generated $\mathrm{H}_{2}$ molecule. Specifically, we derive the equations describing the relationship between the $\mathrm{H}^{-}$beam acceleration voltage and the floating cell voltage $U_{\mathrm{f}}$ necessary to maintain a constant $E_{\mathrm{H}_{2}}$ as we vary $U_{\mathrm{f}}$ (and thereby vary $E_{r}$ ). For our purposes it is sufficient to treat the colliding systems in $1 \mathrm{D}$. Additionally, we ignore the minuscule effects of the $3.75 \mathrm{eV}$ released in the $\mathrm{AD}$ process, an energy which is shared between the detached electron and the internal degrees of freedom of the $\mathrm{H}_{2}$. This energy is insignificant when compared with the $\sim 10 \mathrm{keV}$ energy of the $\mathrm{H}^{-}$and $\mathrm{H}$ beams and the $\sim 20 \mathrm{keV}$ energy of the $\mathrm{H}_{2}$.

Within these approximations, conservation of momentum dictates that the $\mathrm{H}_{2}$ molecule will have a velocity given by

$$
V_{\mathrm{H}_{2}}=\frac{m_{\mathrm{H}^{-}} v_{\mathrm{H}^{-}}+m_{\mathrm{H}} v_{\mathrm{H}}}{M_{\mathrm{H}_{2}}} .
$$

To within the accuracy of our work, we can take $m_{\mathrm{H}^{-}}=m_{\mathrm{H}}$ and $M_{\mathrm{H}_{2}}=2 m_{\mathrm{H}}$ giving

$$
V_{\mathrm{H}_{2}}=\frac{1}{2}\left(v_{\mathrm{H}^{-}}+v_{\mathrm{H}}\right) \text {. }
$$

The $\mathrm{H}_{2}$ laboratory energy is then given by

$$
E_{\mathrm{H}_{2}}=\frac{1}{2} M_{\mathrm{H}_{2}} V_{\mathrm{H}_{2}}^{2}=\frac{m_{\mathrm{H}}}{4}\left(v_{\mathrm{H}^{-}}^{2}+v_{\mathrm{H}}^{2}+2 v_{\mathrm{H}^{-}} v_{\mathrm{H}}\right) \text {. }
$$

The source is operated at a nominal voltage $U_{\mathrm{s}}<0$ with respect to ground. We add to this a voltage of $\Delta$ as described below. The resulting energy and velocity of the $\mathrm{H}^{-}$beam are

$$
E_{\mathrm{H}^{-}}=-e\left(U_{\mathrm{s}}+\Delta\right)
$$

and

$$
v_{\mathrm{H}^{-}}=\sqrt{\frac{-2 e\left(U_{\mathrm{s}}+\Delta\right)}{m_{\mathrm{H}}}} .
$$

The energy of the $\mathrm{H}$ beam is set by changing the effective ground seen by the $\mathrm{H}^{-}$beam in the PD region $\left(U_{\mathrm{f}}\right)$. In this way we can accelerate or decelerate the parent $\mathrm{H}^{-}$beam. Thus we have for the energy and velocity of the $\mathrm{H}$ beam

$$
E_{\mathrm{H}}=-e\left(U_{\mathrm{s}}+\Delta-U_{\mathrm{f}}\right)
$$

and

$$
v_{\mathrm{H}}=\sqrt{\frac{-2 e\left(U_{\mathrm{S}}+\Delta-U_{\mathrm{f}}\right)}{m_{\mathrm{H}}}} .
$$

The relative collision energy $E_{r}$ in the center-of-mass of the colliding $\mathrm{H}^{-}$and $\mathrm{H}$ beams is given by Eq. (2). To control this, we adjust $U_{\mathrm{f}}$. In order to maintain a constant product $\mathrm{H}_{2}$ energy versus $U_{\mathrm{f}}$, we adjust the source voltage by an amount $\Delta$.

Substituting Eqs. (A5) and (A7) into Eq. (A3) we find 


$$
\begin{aligned}
E_{\mathrm{H}_{2}}= & \frac{-e}{2}\left[2 U_{\mathrm{s}}+2 \Delta-U_{\mathrm{f}}\right. \\
& \left.+2 U_{\mathrm{s}} \sqrt{1+\frac{2 \Delta-U_{\mathrm{f}}}{U_{\mathrm{s}}}+\frac{\Delta\left(\Delta-U_{\mathrm{f}}\right)}{U_{\mathrm{s}}^{2}}}\right] .
\end{aligned}
$$

Expanding this to first order gives

$$
E_{\mathrm{H}_{2}} \approx \frac{-e}{2}\left[4 U_{\mathrm{s}}+4 \Delta-2 U_{\mathrm{f}}+\frac{\Delta\left(\Delta-U_{\mathrm{f}}\right)}{U_{\mathrm{s}}}\right] .
$$

In order to maintain $E_{\mathrm{H}_{2}}=-2 e U_{\mathrm{s}}$ we set

$$
4 \Delta-2 U_{\mathrm{f}}+\frac{\Delta\left(\Delta-U_{\mathrm{f}}\right)}{U_{\mathrm{s}}}=0,
$$

which we can re-express as

$$
\Delta^{2}+\left(4 U_{\mathrm{s}}-U_{\mathrm{f}}\right) \Delta-2 U_{\mathrm{s}} U_{\mathrm{f}}=0 .
$$

The roots of this equation can be readily solved using the quadratic formula giving

$$
\Delta=-\frac{\left(4 U_{\mathrm{s}}-U_{\mathrm{f}}\right)}{2} \pm \frac{\left(4 U_{\mathrm{s}}-U_{\mathrm{f}}\right)}{2} \sqrt{1+\frac{8 U_{\mathrm{s}} U_{\mathrm{f}}}{\left(4 U_{\mathrm{s}}-U_{\mathrm{f}}\right)^{2}}} .
$$

It is most convenient to keep $\Delta$ small. So we take the + term. Expanding the square root to first order we have

$$
\Delta \approx \frac{2 U_{\mathrm{s}} U_{\mathrm{f}}}{4 U_{\mathrm{s}}-U_{\mathrm{f}}}=\frac{U_{\mathrm{f}}}{2\left(1-U_{\mathrm{f}} / 4 U_{\mathrm{s}}\right)} .
$$

Again expanding to first order yields

$$
\Delta \approx \frac{U_{\mathrm{f}}}{2}\left(1+\frac{U_{\mathrm{f}}}{4 U_{\mathrm{s}}}\right) .
$$

For typical values of $U_{\mathrm{s}}=-10 \mathrm{keV}$ and $\left|U_{\mathrm{f}}\right|<700 \mathrm{eV}$, the first order correction has less than a $2 \%$ effect on $\Delta$. Second order corrections are expected to be a $₫ 0.03 \%$ effect. So we have used the zeroth order correction $\Delta=U_{\mathrm{f}} / 2$. This gives

$$
E_{\mathrm{H}^{-}}=-e\left(U_{\mathrm{s}}+\frac{U_{\mathrm{f}}}{2}\right)
$$

and

$$
E_{\mathrm{H}}=-e\left(U_{\mathrm{s}}-\frac{U_{\mathrm{f}}}{2}\right) .
$$

Inserting our zeroth order expression for $\Delta$ into Eq. (A8) we find

$$
E_{\mathrm{H}_{2}}=-e\left[U_{\mathrm{s}}+U_{\mathrm{s}} \sqrt{1-\frac{U_{\mathrm{f}}^{2}}{4 U_{\mathrm{s}}^{2}}}\right] .
$$

For the typical values of $U_{\mathrm{s}}$ and $\left|U_{\mathrm{f}}\right|$ given above, the zeroth order correction results in a value of $E_{\mathrm{H}_{2}}$ which is an insignificant $6 \mathrm{eV}$ lower than the desired $E_{\mathrm{H}_{2}}=2 e U_{\mathrm{s}}=20 \mathrm{keV}$. This is well within the energy acceptance of the analyzer.

${ }^{1}$ R. Keller, R. W. Thomae, M. P. Stockli, and R. F. Welton, in High Intensity and High Brightness Hadron Beams, edited by W. Chou, Y. Mori, D. Neuffer, and J.-F. Ostiguy (American Institute of Physics, Melville, 2002), p. 276.

${ }^{2}$ J. G. Alessi, in High Intensity and High Brightness Hadron Beams, edited by W. Chou, Y. Mori, D. Neuffer, and J.-F. Ostiguy (American Institute of Physics, Melville, 2002), p. 279.
${ }^{3}$ J. W. G. Thomason, H. Klein, and J. Peters, in High Intensity and High Brightness Hadron Beams, edited by W. Chou, Y. Mori, D. Neuffer, and J.-F. Ostiguy (American Institute of Physics, Melville, 2002), p. 293.

${ }^{4}$ M. Taniguchi, M. Hanada, T. Iga, T. Inoue, M. Kashiwagi, T. Morista, Y. Okumura, Y. Shimizu, T. Takayanagi, K. Watanabe, and T. Imai, Nucl. Fusion 43, 665 (2003).

${ }^{5}$ O. Kaneko, Y. Takeiri, K. Tsumori, Y. Oka, M. Osakabe, K. Ikeda, K. Nagaoka, T. Kawamoto, E. Asano, and M. Sato, Nucl. Fusion 43, 692 (2003).

${ }^{6}$ J. R. Hiskes, in Production and Neutralization of Negative Ions and Beams, edited by J. G. Alessi and A. Hershcovitch (American Institute of Physics, New York, 1994), p. 155.

${ }^{7}$ M. Capitelli, R. Celiberto, F. Esposito, A. Laricchiuta, K. Hassouni, and S. Longo, Plasma Sources Sci. Technol. 11, A7 (2002).

${ }^{8}$ S. T. Lee and J. M. Farrar, J. Chem. Phys. 111, 7348 (1999).

${ }^{9}$ A. J. Lichtenberg, M. A. Lieberman, I. G. Kouznetsov, and T. H. Chung, Plasma Sources Sci. Technol. 9, 45 (2000).

${ }^{10}$ A. Kono, Appl. Surf. Sci. 192, 115 (2002).

${ }^{11}$ R. N. Franklin, Plasma Sources Sci. Technol. 11, A31 (2002).

${ }^{12}$ J. T. Gudmundsson, I. G. Kouznetsov, K. K. Patel, and M. A. Lieberman, J. Phys. D 34, 1100 (2001).

${ }^{13}$ S.-Y. So, A. Oda, H. Sugawara, and Y. Sakai, J. Phys. D 34, 1919 (2001).

${ }^{14}$ R. N. Franklin, J. Phys. D 34, 1834 (2001).

${ }^{15}$ S. P. Oh and Z. Haiman, Astrophys. J. 569, 558 (2002).

${ }^{16}$ S. L. Lepp, P. C. Stancil, and A. Dalgarno, J. Phys. B 35, R57 (2002).

${ }^{17}$ S. C. O. Glover and D. W. Savin, Mon. Not. R. Astron. Soc. 393, 911 (2009).

${ }^{18}$ S. C. Glover, D. W. Savin, and A.-K. Jappsen, Astrophys. J. 640, 553 (2006).

${ }^{19}$ S. C. O. Glover and T. Abel, Mon. Not. R. Astron. Soc. 388, 1627 (2008).

${ }^{20}$ J. Fritzenwallner and E. Kopp, Adv. Space Res. 21, 891 (1998).

${ }^{21}$ R. P. Wayne, Chemistry of Atmospheres: An Introduction to the Chemistry of the Atmospheres of Earth, the Planets, and Their Satellites (Oxford University Press, Oxford, 2000).

${ }^{22}$ R. W. Schunk and A. F. Nagy, Ionospheres: Physics, Plasma Physics, and Chemistry (Cambridge University Press, Cambridge, 2000).

${ }^{23}$ G. J. Molina-Cuberos, J. J. L'opez-Moreno, R. Rodrigo, H. Lichtenegger, and K. Schwingenschuh, Adv. Space Res. 27, 1801 (2001).

${ }^{24}$ M. C. McCarthy, C. A. Gottlieb, H. Gupta, and P. Thaddeus, Astrophys. J. 652, L141 (2006).

${ }^{25}$ J. Cernicharo, M. Muélin, M. Agúndez, K. Kawaguchi, M. McCarthy, and P. Thaddeus, Astron. Astrophys. 467, L37 (2007).

${ }^{26}$ S. Brünken, H. Gupta, C. A. Gottlieb, M. C. McCarthy, and P. Thaddues, Astrophys. J. 664, L43 (2007).

${ }^{27}$ P. Thaddeus, C. A. Gottlieb, H. Gupta, S. Brünken, M. C. McCarthy, M. Agúndez, M. Guélin, and J. Cernicharo, Astrophys. J. 677, 1132 (2008).

${ }^{28}$ E. Herbst and Y. Osamura, Astrophys. J. 679, 1670 (2008).

${ }^{29}$ V. Wakelam and E. Herbst, Astrophys. J. 680, 371 (2008).

${ }^{30}$ N. Harada and E. Herbst, Astrophys. J. 685, 272 (2008).

${ }^{31}$ A. A. Viggiano and R. A. Morris, J. Phys. Chem. 100, 19227 (1996).

${ }^{32}$ P. G. Wenthold, J. Hu, and R. R. Squires, J. Mass Spectrom. 33, 796 (1998).

${ }^{33}$ V. M. Bierbaum, in The Encyclopedia of Mass Spectrometry: Volume 1-Theory and Ion Chemistry, edited by M. L. Gross and R. Caprioli (Elsevier, Amsterdam, 2003), p. 98.

${ }^{34}$ B. A. Eckenrode, G. L. Glish, and S. A. McLuckey, Int. J. Mass Spectrom. Ion Process. 99, 151 (1990).

${ }^{35}$ E. R. Lovejoy and R. R. Wilson, J. Phys. Chem. A 102, 2309 (1998).

${ }^{36} \mathrm{~J}$. M. Farrar, in The Encyclopedia of Mass Spectrometry: Volume 1-Theory and Ion Chemistry, edited by M. L. Gross and R. Caprioli (Elsevier, Amsterdam, 2003), p. 158.

${ }^{37}$ S. Zivanov, M. Cizek, J. Horacek, and M. Allan, J. Phys. B 36, 3513 (2003).

${ }^{38}$ K. Rempala and K. M. Ervin, J. Chem. Phys. 112, 4579 (2000).

${ }^{39}$ L. A. Angel, M. K. Dogbevia, K. M. Rempala, and K. M. Ervin, J. Chem. Phys. 119, 8996 (2003).

${ }^{40}$ A. Viggiano, personal communication (2004).

${ }^{41}$ A. L. Schmeltekopf, F. C. Fehsenfeld, and E. E. Ferguson, Astrophys. J. 148, L155 (1967).

${ }^{42}$ R. I. Kaiser and A. G. Suits, Rev. Sci. Instrum. 66, 5405 (1995).

${ }^{43}$ I. Savić, I. Čermák, and D. Gerlich, Int. J. Mass Spectrom. 240, 139 (2005).

${ }^{44}$ J. Glosik, I. Korolov, R. Plasil, O. Nototny, T. Kotrik, P. Hlavenka, J. Varju, C. H. Greene, V. Kokoouline, and I. A. Mikhailov, J. Phys. B 41, 
191001 (2008)

${ }^{45}$ R. Otto, J. Mikosch, S. Trippel, M. Weidemüller, and R. Wester, Phys. Rev. Lett. 101, 063201 (2008).

${ }^{46}$ A. Luca, B. Borodi, and D. Gerlich, Proceedings of the 24th International Conference in Photonic, Electronic, and Atomic Collisions, Rosario, Argentina, 20-26 July, 2005 (unpublished).

${ }^{47}$ V. F. DeTuri, P. A. Hintz, and K. M. Ervin, J. Phys. Chem. A 101, 5969 (1999).

${ }^{48}$ E. Haufler, S. Schlemmer, and D. Gerlich, J. Phys. Chem. A 101, 6441 (1997).

${ }^{49}$ R. H. Neynaber, Adv. At. Mol. Phys. 5, 57 (1969)

${ }^{50}$ F. Brouillard, Adv. Mass Spectrom. 5, 153 (1970).

${ }^{51}$ C. C. Havener, in Accelerator-Based Atomic Physics Techniques and Applications, edited by S. M. Shafroth and J. C. Austin (American Institute of Physics, Woodbury, 1997), p. 117.

${ }^{52}$ R. A. Phaneuf, C. C. Havener, G. H. Dunn, and A. M. Müller, Rep. Prog. Phys. 62, 1143 (1999).

${ }^{53}$ C. C. Havener, M. S. Huq, H. F. Krause, P. A. Schulz, and R. A. Phaneuf, Phys. Rev. A 39, 1725 (1989).

${ }^{54}$ T. Nzeyimana, E. A. Naji, X. Urbain, and A. Le Padellec, Eur. Phys. J. D 19, 315 (2002)

${ }^{55}$ J. Horáček, M. Č́̌žek, H. Houfek, P. Kolorenč, and W. Domcke, Phys. Rev. A 70, 052712 (2004).

${ }^{56}$ D. F. Dance, M. F. A. Harrison, and A. C. H. Smith, Proc. R. Soc. London, Ser. A 290, 74 (1966).

${ }^{57}$ F. Brouillard and W. Claeys, in Physics of Ion-Ion and Electron-Ion Collisions, edited by F. Brouillard and J. W. McGowan (Plenum, New York, 1983) Vol. 83, p. 415.

${ }^{58}$ E. M. Purcell, Phys. Rev. 54, 818 (1938).

${ }^{59}$ R. E. Imhof, A. Adams, and G. C. King, J. Phys. E 9, 138 (1976).

${ }^{60}$ D. Q. Hu and K. T. Leung, Rev. Sci. Instrum. 66, 2865 (1995).
${ }^{61}$ T. J. M. Zouros and E. P. Benis, J. Electron. Spectrosc. Relat. Phenom. 125, 221 (2002); T. J. M. Zouros and E. P. Benis, ibid. 142, 175(E) (2005).

${ }^{62}$ T. J. M. Zouros, J. Electron. Spectrosc. Relat. Phenom. 152, 67 (2006); 153, 102(E) (2006).

${ }^{63}$ J. H. Moore, C. C. Davis, and M. A. Coplan, Building Scientific Apparatus: A Practical Guide to Design and Construction (Westview, Boulder, 2003).

${ }^{64}$ www.simion.com.

${ }^{65}$ C. C. Havener, M. A. Haque, A. C. H. Smith, X. Urbain, and P. A. Zeijlmans van Emmichoven, in Sixth International Conference on the Physics of Highly Charged Ions, edited by P. Richard, M. Stockli, C. L. Cocke, and C. D. Lin (AIP, New York, 1993), p. 274.

${ }^{66}$ D. G. Seely, H. Brunhs, D. W. Savin, T. J. Kvale, E. Galutschek, H. Aliabadi, and C. C. Havener, Nucl. Instrum. Methods Phys. Res. A 585, 69 (2008).

${ }^{67}$ V. Ziemann, Vacuum tracking Tech. Rep. SLAC-Pub-5962 (1992) (Stanford, CA: SLAC).

${ }^{68}$ V. Ziemann, Vacuum 81, 866 (2007).

${ }^{69}$ R. Browning, C. J. Latimer, and H. B. Gilbody, J. Phys. B 3, 667 (1970).

${ }^{70}$ H. Kreckel, H. Bruhns, and D. W. Savin, United States Patent Pending.

${ }^{71}$ P. Dahl, Introduction to Electron and Ion Optics (Academic, New York, 1973).

${ }^{72}$ D. H. Crandall, J. A. Ray, and C. Cisneros, Rev. Sci. Instrum. 46, 562 (1975).

${ }^{73}$ D. W. Savin, L. D. Gardner, D. B. Reisenfeld, A. R. Young, and J. L. Kohl, Rev. Sci. Instrum. 66, 67 (1995).

${ }^{74}$ J. D. Thomas, G. S. Hodges, D. G. Seely, N. A. Moroz, and T. J. Kvale, Nucl. Instrum. Methods Phys. Res. A 536, 11 (2005).

${ }^{75}$ H. Tawara, At. Data Nucl. Data Tables 22, 491 (1978).

${ }^{76}$ M. Reiser, Theory and Design of Charged Particle Beams (Wiley, New York, 1994). 